\title{
Tratamiento del Trastorno límite de la personalidad con antagonistas opioides: buprenorfina, nalmefeno, naloxona y naltrexona en el tratamiento de síntomas disociativos, automutilaciones y conducta suicida
}

\author{
María Cecilia García ${ }^{1}$
}

1. Médica Especialista en Psiquiatría. Médica de Planta en Hospital Aeronáutico Central. Jefa de Residentes en Hospital Militar Campo de Mayo. Vicepresidenta del Capítulo de Juego Patológico y otras Adicciones Conductuales de APSA. Miembro permanente en el Capítulo de Psicotrauma de APSA.

Autor correspondiente: María Cecilia García, E-mail: cecigarcia@gmail.com

\begin{abstract}
Resumen
Objetivo: El sistema opioide juega un rol importante en la modulación del ánimo, la recompensa y el dolor, encontrándose inestable en personas con Trastorno Límite de la Personalidad. Analizamos la evidencia sobre la eficacia del uso de fármacos que en alguna medida antagonizan a los opioides, en el tratamiento de síntomas disociativos, automutilaciones y conducta suicida de estos pacientes. Método: Se realizó una revisión sistemática de la evidencia publicada, utilizando los motores de búsqueda MEDLINE y LILACS para identificar los artículos de interés. Fueron incluidos artículos con diseño experimental u observacional, que divulguen alguna medida de impacto en muestras de pacientes límites con síntomas disociativos, automutilaciones y conducta suicida. Resultados: Se incluyó un total de 8 estudios. La evidencia aporta expectativas interesantes sobre posibles líneas de tratamiento del trastorno. El subgrupo que se vio más beneficiado fue el que presentaba analgesia y mayor número de criterios diagnósticos. Conclusión: Se necesitan más estudios de mejor calidad metodológica, en muestras poblacionales más grandes y con control de variables de confusión, que nos permitan realizar una estimación válida para un cálculo de potencia, y así poder arribar a conclusiones más firmes.
\end{abstract}

Palabras claves: Trastorno Límite de la Personalidad - Trastornos disociativos - Conductas autolesivas - Suicidio - Analgesia Buprenorfina - Nalmefeno - Naloxona - Naltrexona. 
TREATMENT OF BORDERLINE PERSONALITY DISORDER WITH OPIOID ANTAGONISTS: BUPRENORPHINE, NALMEFENE, NALOXONE AND NALTREXONE IN THE TREATMENT OF DISSOCIATIVE SYMPTOMS, SELF-MUTILATION AND SUICIDAL BEHAVIOR

\begin{abstract}
Objective: Recenttheory has proposed that a dysfunction of the opioid system modulates mood, reward and pain; seems to be unstable in people with Borderline Personality Disorder. Our purpose is analyze the evidence on the efficacy of the use of buprenorphine, nalmefene, naloxone and naltrexone, in the treatment of dissociative symptoms, self-mutilation and suicidal behavior of these patients. Method: We conducted a systematic search of MEDLINE and LILACS databases, to retriever elevant articles. Included studies were experimental and observational design of borderline personality samples in which dissociative symptoms, selfmutilation or suicidal behaviour was reported as an outcome and evaluated with some measure of impact. Results: A total of 8 studies were reviewed. These provided interesting expectations about posible treatment lines in Borderline Personality Disorder using opioid antagonists. The subgroup most benefited was the one who has analgesia and highest number of diagnostic criteria. Conclusions: Studies of higher methodological quality are needed, in larger population samples and using control of confounding variables that allow us to estimate a value power calculation, and thus be able to support firm conclusions.
\end{abstract}

Keywords: Borderline personality disorder - Dissociative disorders - Selfmutilation - Suicide - Bruprenorphine - Nalmefene Naloxone - Naltrexone.

\section{Introducción}

El sistema opioide ha sido relacionado con la modulación del ánimo, la recompensa y el dolor, y mediaría algunas características del Trastorno Límite (TLP), especialmente en los fenotipos más propensos a automutilarse y a abusar de sustancias (1-3). En ellos la actividad endopioide se encontraría inestable, hallándose en alta o en baja en diferentes momentos en el mismo paciente. Esto nos hace pensar a sus receptores como dianas terapéuticas interesantes (1) pues podrían ser efectivos ante situaciones con componentes afectivos y emocionales específicos (4). Sus potenciales efectos beneficiosos podrían deberse a: a) antagonismo MOR, reduciendo la analgesia asociada al estrés, bloqueando la activación artificial "en modo supervivencia" ante conductas de riesgo o autolesiones, reduciendo la liberación de dopamina en el núcleo accumbens y estabilizando con su administración continuada los niveles de opioides endógenos; b) antagonismo KOR, reduciendo disforia, estados disociativos y produciendo un efecto antidepresivo; pudiendo atenuar la ingesta de alcohol y sustancias psicoactivas.

Los fármacos buprenorfina, nalmefeno, naloxona y naltrexona podrían ser efectivos en el tratamiento de conductas autolesivas no suicidas (NSSI), suicidas y síntomas disociativos en pacientes límite. Indagamos en su posible utilidad analizando la evidencia publicada.

\section{Objetivos}

Primario: analizar la evidencia sobre de la eficacia del uso de buprenorfina, nalmefeno, naloxona y naltrexona en el tratamiento de automutilaciones, conducta suicida y síntomas disociativos de los pacientes con TLP.

Secundario: identificar el perfil de pacientes con mejor respuesta a estos fármacos.

\section{Metodología}

Para responder estos interrogantes se realizó una revi- sión sistemática de la evidencia publicada hasta mayo del 2019, utilizando los motores de búsqueda MEDLINE y LILACS para identificar los artículos de interés. Las búsquedas se realizaron utilizando los siguientes ítems: "borderline personality disorder" $[\mathrm{MeSH}]$; más uno de los siguientes: "dissociative disorders" [MeSH], "dissociative", "dissociation", "selfmutilation" [MeSH], "self injuries", "injury", "wounds and injuries" [MeSH], "self-injurious behaviour" [MeSH], "self injurious", "self-mutilation disorder", "suicid*" [All Fields] y uno de los siguientes: "buprenorphine" [MeSH], "nalmefene" [MeSH], "naloxone" [MeSH] y "naltrexone" [MeSH]. Fueron consideradas todas las investigaciones que reunieron los criterios de inclusión, estos fueron: 1) artículo original; 2) diseño experimental u observacional; 3) diagnóstico de TLP; 4) que divulgue alguna medida de impacto en relación a nuestros objetivos. Los criterios de exclusión fueron: 1) estudio duplicado; 2) reporte de casos clínicos; 3) revisión; 4) que no analice la relación entre los fármacos que antagonizan al sistema opioide y las conductas autolesivas, suicidas o síntomas disociativos; 5) personas con trastornos del neurodesarrollo o psicóticos. De los artículos seleccionados, se revisaron adicionalmente referencias y publicaciones citadas para identificar cualquier documentación adicional de relevancia.

\section{Resultados}

La búsqueda inicial arrojó 21 resultados de los cuáles se eliminaron aquellos que no cumplían los criterios de inclusión. Fueron eliminados 4 artículos por tratarse de revisiones, 4 por tratarse de reportes de casos clínicos, 3 estudios cuyas medidas de impacto no se condecían con nuestros objetivos y 2 estudios duplicados.

Finalmente, se incluyeron 8 estudios en esta revisión con un total de 187 pacientes tratados con estos fármacos (Figura 1). En general, los estudios fueron realizados en población femenina, presentando un reducido tamaño muestral. El tiempo de duración promedio fue de 6 semanas, excepto uno de los ensayos que duró 3 días (5). 
Figura 1. Diagrama de flujo de la búsqueda sistemática de la evidencia publicada en la literatura respecto al tratamiento de conductas autolesivas con antagonistas opioides, 2019.

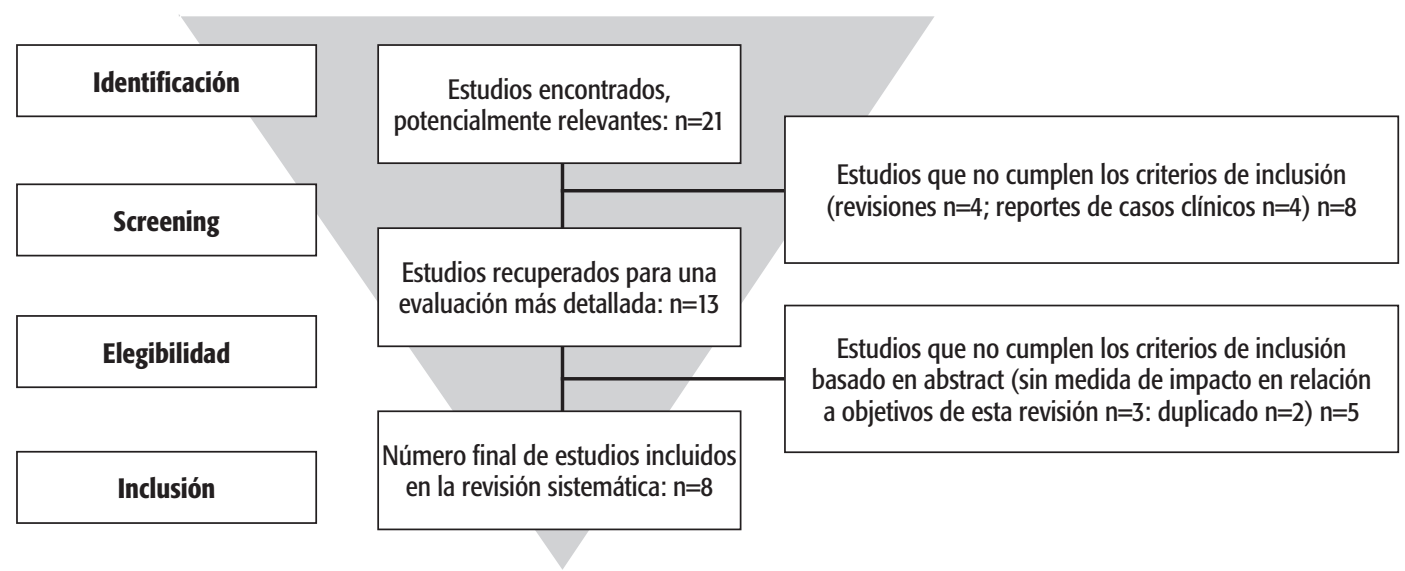

La Tabla 1 describe los 8 artículos seleccionados en esta revisión de la literatura. Dos ensayos utilizaron naloxona (NLX); cuatro, naltrexona (NTX); uno, bupre- norfina (BPF) y otro, nalmefeno (NMF). La mitad de los estudios utilizó comparador con placebo, siendo dos de ellos de asignación cruzada.

Tabla 1. Autores, Año de Publicación, País, Grupo Diagnóstico, Características de la Muestra, Tratamiento, Comparador, Duración, Diseño, Medidas Clínicas Evaluadas y Hallazgos clave de ocho estudios incluidos en esta revisión sobre tratamiento con fármacos que antagonizan el sistema opioide en el TLP, 2019

\begin{tabular}{|c|c|c|c|c|c|c|c|c|c|c|}
\hline Autor & País & Grupo & Muestra & Mujeres & Tratamiento & Comparador & Duración & Diseño & $\begin{array}{c}\text { Medidas clínicas/ } \\
\text { Escalas }\end{array}$ & Hallazgos clave \\
\hline $\begin{array}{l}\text { Russ } \\
(1994)\end{array}$ & EEUU & TLP & $\begin{array}{c}\mathrm{N}=11 \\
19 \text { a } 41 \\
\text { años }\end{array}$ & $100 \%$ & $\begin{array}{l}\text { NLX EV } \\
2 \mathrm{mg} / \text { día }\end{array}$ & $\begin{array}{l}\text { Placebo } \\
\text { (solución } \\
\text { salina) }\end{array}$ & 3 días & $\begin{array}{l}\text { Ensayo clínico } \\
\text { aleatorizado, } \\
\text { doble ciego, } \\
\text { controlado }\end{array}$ & $\begin{array}{c}\text { Perfil de los } \\
\text { estados de } \\
\text { ánimo (POMS), } \\
\text { Escala propia de } \\
\text { intensidad del } \\
\text { dolor y malestar. }\end{array}$ & $\begin{array}{l}\text { El grupo TLP con analgesia } \\
\text { ante NSSI, experimentó más } \\
\text { dolor después de NLX. POMS } \\
\text { no disminuyó, pero ante la } \\
\text { aplicación de un estímulo } \\
\text { doloroso mejoraron las } \\
\text { puntuaciones de depresión } \\
{[\mathrm{F}(4,36)=2,88, \mathrm{p}=0,04] \text { y }} \\
\text { tensión/malestar }[\mathrm{F}(4,36)= \\
3,42, \mathrm{p}=0,02] \text {. NLX atenuó la } \\
\text { mejoría de la disforia post NSSI. }\end{array}$ \\
\hline $\begin{array}{l}\text { Sonne } \\
\text { (1996) }\end{array}$ & EEUU & TLP & $\begin{array}{c}\mathrm{N}=5 \\
26 \text { a } 36 \\
\text { años }\end{array}$ & $100 \%$ & $\begin{array}{c}\text { NTX VO } \\
50-100 \mathrm{mg} / \text { día }\end{array}$ & Ninguno & $\begin{array}{c}3 \\
\text { semanas }\end{array}$ & $\begin{array}{l}\text { Ensayo clínico } \\
\text { no controlado, } \\
\text { no ciego }\end{array}$ & $\begin{array}{c}\text { Escala de Yale- } \\
\text { Brown para } \\
\text { trastornos } \\
\text { obsesivos } \\
\text { compulsivos } \\
\text { (YBOCS) } \\
\text { modificada para } \\
\text { ideas obsesivas } \\
\text { NSSI. }\end{array}$ & $\begin{array}{l}\text { El 80\% dejó de autolesionarse, } \\
\text { disminuyendo: NSSI/día 60\% } \\
\text { (x: S1 28\%, S2 11\%, S3 26\%), } \\
\text { NSSI }[(\mathrm{S} 12,0, \mathrm{~S} 20,4, \mathrm{S3} 1,8 \%) \text {, } \\
(\mathrm{t}=4,0, \mathrm{p}<0,05)] \text {, pensamientos } \\
\mathrm{NSSI} / \text { día en S2 comparada } \\
\text { con S3 (t_2,16, p<0,05) y } \\
\text { de S2 comparada con S1 } \\
(\mathrm{t}=2,37, \mathrm{p}<0,05) \text {, aumento en } \\
\mathrm{S} 3 \text { ( } \mathrm{t}=6,07, \mathrm{p}<0,05) \text {. YBOCS } \\
\text { disminuyó (t=3,69, p>0,0,5). Al } \\
\text { discontinuar NXT, aumentaron } \\
\text { NSSI e YBOCS. Sugiere rebote. }\end{array}$ \\
\hline $\begin{array}{l}\text { Roth } \\
\text { (1996) }\end{array}$ & EEUU & $\begin{array}{c}\text { NSSI } \\
\text { en TLP } \\
\text { y otros } \\
\text { trastornos }\end{array}$ & $\begin{array}{l}\mathrm{N}=7, \\
18 \text { a } 50 \\
\text { años }\end{array}$ & $100 \%$ & $\begin{array}{c}\text { NTX VO } \\
50 \mathrm{mg} / \text { día }\end{array}$ & Ninguno & $\begin{array}{c}\text { Media } \\
10,7 \\
\text { semanas }\end{array}$ & $\begin{array}{c}\text { Ensayo abierto, } \\
\text { no controlado, } \\
\text { no ciego }\end{array}$ & $\begin{array}{c}\text { Escala propia } \\
\text { evaluando NSSI, } \\
\text { analgesia, disforia, } \\
\text { tensión interna. }\end{array}$ & $\begin{array}{l}\text { En todos los pacientes } \\
\text { disminuyeron NSSI, analgesia } \\
\text { y disforia. El } 85,7 \% \text { de } \\
\text { los pacientes dejaron de } \\
\text { autolesionarse. Al suspender } \\
\text { NTX 42.8\% reanudó NSSI. }\end{array}$ \\
\hline
\end{tabular}




\begin{tabular}{|c|c|c|c|c|c|c|c|c|c|c|}
\hline Autor & País & Grupo & Muestra & Mujeres & Tratamiento & Comparador & Duración & Diseño & $\begin{array}{l}\text { Medidas clínicas/ } \\
\text { Escalas }\end{array}$ & Hallazgos clave \\
\hline $\begin{array}{l}\text { Bohus } \\
\text { (1999) }\end{array}$ & Alemania & TLP & $\begin{array}{c}\mathrm{N}=13 \\
17 \text { a } 47 \\
\text { años }\end{array}$ & $100 \%$ & $\begin{array}{c}\text { NTX VO } \\
\text { 25-100 mg/día }\end{array}$ & Ninguno & $\begin{array}{c}2 \\
\text { semanas }\end{array}$ & $\begin{array}{c}\text { Ensayo clínico } \\
\text { abierto, no } \\
\text { controlado. } \\
\text { Dos grupos, } \\
\text { superposición } \\
\text { de } 5 \text { pacientes }\end{array}$ & $\begin{array}{c}\text { Cuestionario } \\
\text { autoadministrado } \\
\text { evaluando } \\
\text { disociación, } \\
\text { analgesia, tensión } \\
\text { e inmovilidad } \\
\text { (DAISS, } \\
\text { no publicada), } \\
\text { flashbacks. }\end{array}$ & 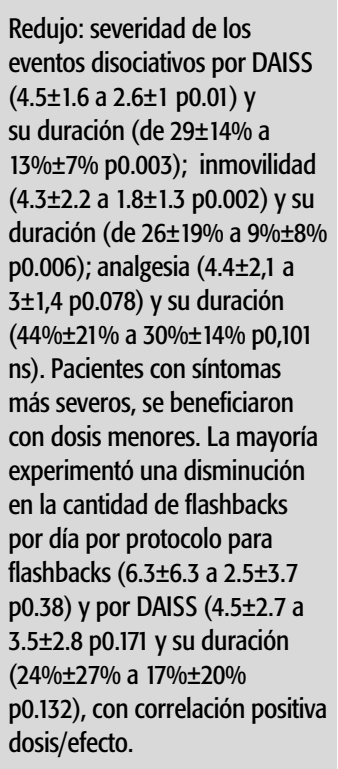 \\
\hline $\begin{array}{c}\text { Philipsen } \\
\text { (2004) }\end{array}$ & Alemania & TLP & $\begin{array}{c}\mathrm{N}=9, \\
25 \text { a } 43 \\
\text { años }\end{array}$ & $100 \%$ & $\begin{array}{c}\text { NLX EV } \\
0,4 \text { mg } \\
\text { tras evento } \\
\text { disociativo }\end{array}$ & $\begin{array}{l}\text { Placebo } \\
\text { EV }\end{array}$ & $\begin{array}{c}6 \\
\text { semanas }\end{array}$ & $\begin{array}{c}\text { Ensayo clínico } \\
\text { controlado, } \\
\text { aleatorizado, } \\
\text { doble ciego, } \\
\text { cruzado }\end{array}$ & \begin{tabular}{|c|} 
Escala de Estados \\
Disociativos \\
(CADSS), de \\
autoevaluación \\
(DSS), de \\
experiencias \\
disociativas (DES) \\
y de disociación \\
somatomorfa \\
(SDQ-20), \\
Inventario de \\
depresión de Beck \\
y Hamilton.
\end{tabular} & $\begin{array}{l}\text { No fue superior a placebo en } \\
\text { estados disociativos agudos. } \\
\text { Correlación entre criterios para } \\
\text { TLP y mejoría sintomática. } \\
44 \% \text { respondió a NLX, de los } \\
\text { cuáles el } 25 \% \text { también lo hizo } \\
\text { a placebo, } 22 \% \text { respondió } \\
\text { sólo a placebo. Severidad de } \\
\text { los sintomas fue significativa- } \\
\text { mente menor tras la inyección } \\
\text { (F1.8=10.5, p<0.05 para DSS y } \\
\text { F1.8=21.2, p<0.01 para CADSS), } \\
\text { lo cual podría implicar un } \\
\text { efecto antidisociativo de la vía } \\
\text { inyectable. }\end{array}$ \\
\hline $\begin{array}{c}\text { Schmahl } \\
(2012)\end{array}$ & Alemania & TLP & $\begin{array}{c}\mathrm{N}=29 \\
18 \text { a } 44 \\
\text { años }\end{array}$ & $100 \%$ & $\begin{array}{c}\text { NTX VO } \\
50-200 \mathrm{mg} / \mathrm{dia}\end{array}$ & Placebo & $\begin{array}{c}8 \\
\text { semanas }\end{array}$ & $\begin{array}{l}2 \text { ensayos } \\
\text { controlados, } \\
\text { aleatorizados } \\
\text { doble ciego, } \\
\text { cruzado }\end{array}$ & $\begin{array}{c}\text { Escala de } \\
\text { experiencias } \\
\text { de disociación, } \\
\text { de ansiedad de } \\
\text { Hamilton, de } \\
\text { depresión de } \\
\text { Beck y Hamilton, } \\
\text { BSL-23. }\end{array}$ & $\begin{array}{l}\text { En ambos ensayos, los datos } \\
\text { fueron algo a favor de la NTX } \\
\text { con respecto a la intensidad } \\
\text { y la duración de los síntomas } \\
\text { disociativos, que fueron más } \\
\text { altos con placebo (en estudio } \\
1, d=0,13 \text { y } 0,09 \text {; en estudio } \\
2, d=0,41 \text { y } 0,24 \text {, tamaño del } \\
\text { efecto en intensidad y duración, } \\
\text { respectivamente). }\end{array}$ \\
\hline $\begin{array}{l}\text { Yovell } \\
\text { (2016) }\end{array}$ & Israel & $\begin{array}{c}\text { Ideación } \\
\text { Suicida en } \\
\text { TLP } \\
\text { y otros }\end{array}$ & $\begin{array}{c}\mathrm{N}=88 \\
18 \text { a } 65 \\
\text { años } \\
\text { TLP N=34 }\end{array}$ & $72,2 \%$ & $\begin{array}{c}\mathrm{BPF} \\
\mathrm{SL} \\
0,8 \mathrm{mg} / \text { día }\end{array}$ & Placebo & $\begin{array}{c}4 \\
\text { semanas }\end{array}$ & $\begin{array}{c}\text { Ensayo clínico } \\
\text { controlado } \\
\text { aleatorizado }\end{array}$ & \begin{tabular}{|c|} 
Escala de \\
Beck ideación \\
suicida (BSSI), \\
de Depresión \\
II de Beck, de \\
probabilidad de \\
riesgo suicida \\
(SPS), Sufrimiento \\
(item 25).
\end{tabular} & $\begin{array}{l}\text { Disminuyó Ideación Suicida } \\
\text { de Beck semana } 2 \text { (diferencia } \\
\text { media=24.3, } 95 \% \text { IC }=28,5,20,2 ; \\
\mathrm{p}=0.04 \text { ) y al final de la semana } \\
4 \text { (diferencia media=27.1, IC del } \\
95 \%=212.0,22.3 ; \mathrm{p}=0.004) ; \\
\text { SPS diferencia media }=210.4, \text { IC } \\
95 \%=219.7,21.0 ; \mathrm{p}=0.03) \text {. Item } \\
25 \text { (diferencia media=20.8, IC } \\
95 \%=21.5,20.7 ; \mathrm{p}=0.03) .\end{array}$ \\
\hline $\begin{array}{c}\text { Martín- } \\
\text { Blanco } \\
(2017)\end{array}$ & España & TLP & $\begin{array}{l}\mathrm{N}=25 \\
\text { media } \\
34.60 \\
\text { años }\end{array}$ & $93 \%$ & $\begin{array}{c}\text { NMF } \\
\text { Vo } 18 \mathrm{mg} / \text { día }\end{array}$ & Ninguno & $\begin{array}{c}8 \\
\text { semanas }\end{array}$ & $\begin{array}{c}\text { Ensayo abierto, } \\
\text { no controlado, } \\
\text { no ciego }\end{array}$ & $\begin{array}{c}\text { Inventario } \\
\text { de síntomas } \\
\text { borderline } \\
\text { (BSL-23), } \\
\text { Impresión clínica } \\
\text { global (ICG-TLP). }\end{array}$ & $\begin{array}{l}\text { Redujo el consumo de alcohol } \\
\text { y escalas clínicas del TLP. Todos } \\
\text { los dominios sintomáticos } \\
\text { mostraron mejora, excepto la } \\
\text { ideación paranoide. BSL-23 } \\
\text { semana 0: } 54.25 \text {; semana } 8 \text { : } \\
\text { 42.95; ICG-TLP semana } 0: 4.80 \text {; } \\
\text { semana 8: } 3.25 \text {. }\end{array}$ \\
\hline
\end{tabular}




\section{Análisis por fármacos}

La Tabla 2 resume sus características principales.

Tabla 2. Fármacos antagonistas opioides con sus características principales (receptores a los que se acoplan, vía de administración y dosis posible para TLP [SL, sublingual, EV, endonvenosa; IM, intramuscular, SC, subcutánea], metabolismo, unión a proteínas (UP), indicaciones habituales (en gris) e indicaciones posibles para TLP (en negrita)

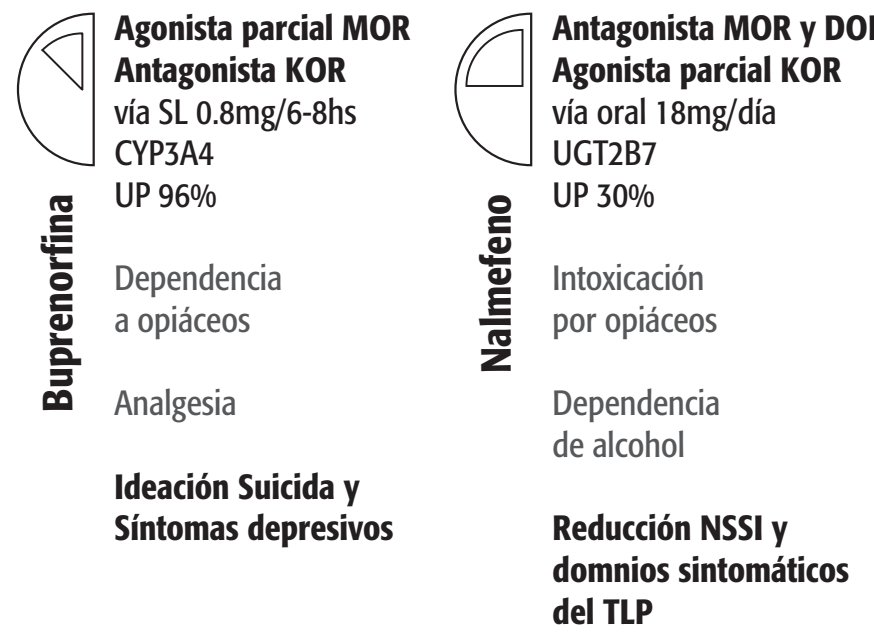

Buprenorfina

Tiene un perfil farmacodinámico complejo: es agonista parcial MOR y potente antagonista KOR. A su vez, sus propiedades dependen de la dosis y vía de administración (6). Podría ser eficaz en pacientes deprimidos y suicidas. Su acción parece adaptarse mejor al perfil del subgrupo TLP con síntomas afectivos relacionados con sentimientos dolorosos de abandono y rechazo. A dosis bajas sería menos activo sobre síntomas neurovegetativos y otros relacionados con la disminución del tono hedónico; además, atenuaría la hiperactivación del sistema pánico endorfinérgico $(7,8)$.

En un ensayo clínico controlado (7) $(n=88)$ se obtuvo una reducción significativa de la ideación suicida grave, 4 veces mayor que con placebo. Esta diferencia se acentuó con el transcurso del tratamiento (mayor a las 4 semanas). La respuesta de la ideación suicida del TLP fue un 20\% mayor que la de sujetos melancólicos.

\section{Nalmefeno}

Es un modulador selectivo del sistema opioide: antagonista MOR y DOR, y agonista parcial KOR (9). En el TLP podría reducir la hipersensibilidad del sistema

Antagonista MOR,
DOR, KOR
vía EV, IM, SC
0,4mg/dosis
Citosólico
UP 32\% a 42\%
por opiáceos
Prueba previa
a naltrexona

\section{Disminución de analgesia y NSSI}

\author{
Antagonista MOR, \\ DOR, KOR \\ vía oral $50 \mathrm{mg} /$ día \\ Citosólico \\ UP $21 \%$ a $28 \%$ \\ Dependencia a \\ opiáceos y alcohol

Disminución
de síntomas
disociativos
y NSSI

opioide ante NSSI bloqueando la estimulación del circuito de refuerzo y recompensa. Además, podría disminuir la gravedad de la sintomatología clínica del TLP en todas sus dimensiones, excepto en ideación paranoide.

En un ensayo clínico abierto $(n=25)$ (10) se obtuvo una reducción significativa del consumo de alcohol, con mejoría de todos los dominios sintomáticos medidos por BSL-23 y ICG-TLP: descenso significativo del $21 \%$ de la puntuación global de BSL-23, del 32.3\% de la ICG-TLP y del $68 \%$ en la escala de consumo excesivo de alcohol (HDD), del 78,5\% para NSSI, del 38\% en la impulsividad, 33\% en la ira e inestabilidad afectiva, 30\% en la sensación de vacío, 25\% la ideación suicida, 20\% la sensación de abandono y de $15.5 \%$ la puntuación para alteraciones de la identidad.

\section{Naloxona}

Es un antagonista opiáceo competitivo inespecífico de receptores MOR, DOR y KOR, por lo cual dificulta la actividad de los opioides. Podría reducir hipersensibilidad del sistema opioide ante NSSI. Disminuiría la analgesia relacionada con el estrés en pacientes que la presentan, lo cual contribuiría a limitar la producción de autolesiones. Se estima que habría mayor respuesta 
mientras más criterios para TLP cumplan los pacientes.

Un estudio (5) la utilizó en dosis de $2 \mathrm{mg}$ EV ( $\mathrm{n}=11)$ obteniendo una reducción numérica pero no significativa de la analgesia en los pacientes con NSSI. Otro ensayo (n=9) (11) comparó 0.4mg EV de NLX con placebo, observando una reducción numérica pero no significativa de eventos disociativos agudos.

\section{Naltrexona}

Es un antagonista opiáceo competitivo inespecífico de receptores MOR, DOR y KOR, dificultando la actividad de los opioides. Podría ser eficaz para síntomas disociativos, reduciendo la cantidad de eventos disociativos por día, su intensidad, duración y la inmovilidad o parálisis; la cantidad de flashbacks al día, su intensidad y duración; y la analgesia relacionada con el estrés. Además, podría ser eficaz en NSSI al disminuir la hipersensibilidad del sistema opioide y, como consecuencia, las conductas por sí mismas y su refuerzo.

Quienes se verían más beneficiados son los pacientes con más criterios diagnósticos, aún en dosis de $50 \mathrm{mg} /$ día. Su administración oral de manera continua sería más eficaz que la aplicación EV o SC de NLX en agudo. Además, es probable un efecto rebote al suspenderla abruptamente.

Un estudio ( $n=13)$ (12) observó una reducción significativa del 50\% en la cantidad de eventos disociativos por día, $42 \%$ en su intensidad y $58 \%$ en la parálisis asociada a estos. Si bien, para otra investigación $(n=29)(13)$ el descenso no fue significativo (aunque sí numérico) de $31 \%$ en la intensidad de los eventos y $41 \%$ en la parálisis asociada.

También redujo la frecuencia de flashbacks un $60 \%$ respecto a la cantidad de episodios, $22 \%$ en su intensidad y $29 \%$ en su duración $(n=13)$ (12). Si bien en otro ensayo estas diferencias fueron menores y no significativas, del 11\% en cantidad y del 13\% en su duración (13).

Respecto a la analgesia relacionada con el estrés, un estudio observó que todos los pacientes experimentaron dolor ante la intervención con NTX $(n=7)$, y una reducción no significativa de 50\% su intensidad y 30\% su duración $(\mathrm{n}=13)(12)$.

Un ensayo $(n=5)$ (14) observó una disminución significativa de $50 \%$ en las ideas NSSI, se redujeron 3 veces los actos y conductas de automutilación (un 75\%), el $80 \%$ de los pacientes dejaron de hacerlo mientras duró la intervención, y el $20 \%$ restante redujo al $60 \%$ su cantidad. Otro (15) observó que el $87,5 \%$ de los pacientes dejaron de autolesionarse, reduciendo además el alivio emocional ante las NSSI ( $\mathrm{n}=7)$. En otro ensayo (13) estas diferencias no fueron significativas pero sí numéricas $(n=29)$. Un estudio observó una diferencia significativa de la disforia ( $\mathrm{n}=7)(15)$, si bien otro halló una reducción numérica pero no significativa $(\mathrm{n}=29)(13)$.

\section{Análisis orientado a los síntomas}

Estos resultados se esquematizan sintéticamente en la Tabla 3.

\section{Tabla 3}

\author{
Síntomas disociativos \\ $\downarrow$ eventos disociativos (intensidad y duración) \\ $\downarrow$ inmovilidad \\ $\downarrow$ flashbacks \\ $\downarrow$ analgesia
}

Más beneficio cuanto mayor es la sintomatología

Más beneficio mientras más criterios diagnósticos

\section{Autolesiones \\ $\downarrow$ cantidad de NSSI \\ $\downarrow$ pensamientos NSSI \\ Más beneficiado quien cumpla más criterios diagnósticos}

\author{
Ideación suicida \\ $\downarrow$ de escala SPS \\ Más efectivo contra ideación suicida del límite que en \\ melancólicos
}

\section{Síntomas depresivos \\ $\downarrow$ de puntuación de Escala de Beck \\ $\downarrow$ de desesperanza}

\section{Síntomas disociativos}

Se correlacionan con experiencias de tensión interna aversiva y conductas autolesivas no suicidas. De hecho, los pacientes utilizan las NSSI empíricamente para finalizar las experiencias disociativas. Los antagonistas opioides completos han demostrado resultados interesantes en el tratamiento de los síntomas disociativos. Se ha observado que NLX y, específicamente NTX, podrían ser eficaces en el control de eventos de despersonalización y desrealización. Todos los ensayos clínicos que han intentado valorar su eficacia, han obtenido reducción en esta sintomatología. Si bien no ha sido posible arribar a conclusiones firmes los resultados son sugerentes:

- Reducción en la cantidad de eventos disociativos por día, su intensidad, duración y la parálisis o inmovilidad tónica. Un estudio $(n=13)$ (12) observó que a partir del tercer día todos los pacientes redujeron significativamente los eventos disociativos: $42 \%$ su intensidad, 55\% 
su duración, 50\% la cantidad de eventos reportados por día y 58\% la parálisis. En esta línea, otros investigadores (13) diseñaron un ensayo clínico con mayor rigurosidad $(n=29)$ controlando confundidores como la reducción espontánea de los síntomas disociativos a lo largo del tiempo y el efecto placebo, obteniendo resultados algo a favor de la NTX respecto a su intensidad y duración (pero en menor medida que los estudios previos). Observaron una reducción numérica no significativa del 31\% y del $41 \%$ respectivamente. Con NLX administrada en dosis única $(n=9)$ no se observaron diferencias significativas respecto a la reducción de eventos disociativos (44\%) comparándola con placebo (33\%) (11). En este ensayo, la severidad de la sintomatología fue significativamente menor ante ambas intervenciones sugiriendo que esto podría deberse al efecto de la aplicación del inyectable (la experiencia dolorosa podría interrumpir el estado disociativo, actuando como las NSSI).

- Reducción en la cantidad de flashbacks al día, su intensidad y duración. Un estudio $(n=13)$ obtuvo una disminución significativa del $60 \%$ en la cantidad de episodios por día, 22\% su intensidad y 29\% su duración, con una correlación positiva dosis/efecto (12). En otro ensayo $(n=29)$ (13) ésta fue menor (11\% en la cantidad y13\% su duración).

- Reducción en la analgesia relacionada con el estrés, impactando sobre las NSSI ya que, al intentar autolesionarse, sentirían dolor y detendrían su conducta. Un estudio ( $\mathrm{n}=11$ ) (5) comprobó que los pacientes con analgesia, la redujeron en un 14\% tras la intervención con NLX. Con NTX se obtuvieron resultados similares: los 7 pacientes que se incluyeron en un estudio (15) experimentaron dolor a partir de su administración. En otro $(n=13)$ la analgesia se redujo en menor medida (50\% su intensidad, 30\% su duración) (12).

- El impacto en las alteraciones sobre la autopercepción no están del todo claras, y deberían ser estudiadas con especificidad. El NMF redujo las alteraciones de la identidad en pacientes TLP $(n=25)$ medidos por ICG-TLP siendo más notoria a las 8 semanas de tratamiento (10).

- El beneficio sería directamente proporcional a la severidad del TLP siendo mayor en los pacientes que cumplan más criterios diagnósticos por DSM $(11,12)$.

- Sería más eficaz la administración oral continuada que en agudo $(11,12)$.

\section{Conductas autolesivas no suicidas}

El mecanismo por el cual disminuirían las NSSI sería doble: debido a la reducción de la analgesia durante la automutilación, eliminando además el alivio de la tensión interna y la disforia, desarticulando el sistema de refuerzo y recompensa mediado por opioides y dopamina. Estos serían:

- Reducción en las NSSI, disminuyendo la hipersensibilidad de los opioides a estas conductas y, por lo tanto, su refuerzo negativo. En un estudio $(n=5)$ el $80 \%$ de los pacientes dejaron de autolesionarse y el 20\% restante disminuyó un 60\% la cantidad de días que lo hicieron, lo cual fue más notorio con la administración continuada de NTX (14). Resultados similares obtuvo otro (15) en el cual disminuyó la frecuencia de NSSI $(n=7)$, el 85,7\% de los pacientes dejaron de autolesionarse mientras duró la intervención, reduciendo además el alivio emocional ante estas conductas. Sin embargo, un ensayo que tomó las NSSI como objetivo secundario a la intervención con NTX, no observó diferencias significativas en comparación con placebo $(n=29)$ (13). Muchos sujetos que presentaban NSSI antes o después de los episodios disociativos, al reducirse éstos con la administración de fármacos, redujeron también las autolesiones (11). Existen, además, algunos reportes de casos que expresan resultados positivos en pacientes TLP con estas conductas (16-18).

El NMF disminuyó significativamente las NSSI $(\mathrm{n}=25)$ en un $78.5 \%$ y la impulsividad en un $38 \%(10)$ medidas por BSL-23 e ICG-TLP, siendo más notorio a la 8va semana de tratamiento.

- Reducción en la cantidad de pensamientos vinculados a autolesionarse, impactando secundariamente en el número y frecuencia de NSSI. Tras la administración de NTX $(n=5)$ disminuyeron a la mitad los pensamientos de automutilación (medido por YBOCS modificada para ideas obsesivas y automutilación) (14).

- Pacientes con más criterios diagnósticos, se verían más beneficiados al utilizar NTX, en dosis de $50 \mathrm{mg} /$ día. La administración continua de NTX sería más eficaz que la aplicación de NLX en agudo.

- Se estima un efecto rebote al suspender abruptamente NTX, por lo que su discontinuación debería ser gradual (especialmente en pacientes que se autolesionan). Un estudio $(n=5)(14)$, evidenció un aumento de 6.7 veces el número de pensamientos NSSI y en otro $(n=9)(15)$ quienes que suspendieron la medicación $(n=2)$ se autolesionaron.

- Podría existir un confundidor ante la intervención con fármacos que utilicen la vía EV, IM o SC para su administración. Los inyectables podrían resultar en una situación que, al generar dolor, active la vía de los opioides endógenos, disminuyendo por sí misma las NSSI y los síntomas disociativos $(\mathrm{n}=9)$ (11).

\section{Ideación suicida}

En el TLP el efecto de reducción en la ideación suicida podría deberse a una atenuación del sistema pánico endorfinérgico $(7,8,19)$. De los estudios que evaluaron esta dimensión, resulta:

Reducción en la ideación suicida con BPF a dosis bajas de $0.8 \mathrm{mg}$ /día SL ( $\mathrm{n}=88$ ) (7). Disminución significativa en la ideación suicida grave medida por SPS (4 veces mayor que con placebo), diferencia que se incrementó a favor de esta intervención hacia el final de la 4ta semana de tratamiento. Sería más efectivo contra la ideación suicida atípica de los pacientes límite que para la de sujetos melancólicos, con un índice de respuesta un 20\% mayor. Además, obtuvo una reducción numérica (no significativa) de la Escala de Beck para suicidio. 
El NMF redujo en un 25\% la ideación suicida en pacientes límite $(\mathrm{n}=25)$ medido por ICG-TLP, siendo esta diferencia significativa y más notoria a medida que avanzó el tratamiento (10).

\section{Síntomas depresivos}

Al utilizar BPF como intervención en 88 pacientes, se obtuvo un descenso en la Escala de Depresión de Beck y en el grado de desesperanza, si bien estas diferencias no fueron significativas al compararlas con placebo. Pero cuando esto se evaluó a través de la Escala de Ideación Suicida de Beck y la pregunta 25 sobre sufrimiento subjetivo de la SPS, se plasmó una diferencia significativa a favor de la eficacia de la BPF sobre placebo (7).

No se observaron diferencias significativas en las puntuaciones para depresión medidas por POMS con NLX (5); tampoco en las escalas de Depresión de Beck ni de Hamilton. Un estudio $(n=7)$ observó una disminución de la disforia con NTX, si bien no la cuantificó (15); en otro $(n=29)$ no evidenciaron diferencias significativas al tomar las escalas de Depresión de Hamilton y Beck (13).

\section{Evaluación de calidad de los estudios}

Para evaluar la calidad de los estudios, se realizó inicialmente la escala de Jadad por su sencillez y practicidad, siendo sus parámetros de valoración: asignación aleatoria con métodos adecuados, enmascaramiento y descripción o no de las pérdidas y retiradas del estudio. Se aplicó a todos los ensayos, siendo 4 estudios de buena calidad con puntuación mayor a 3 y 4 de baja calidad. Se mensuran los resultados en la Tabla 4.

Además, se trabajó con las "Recomendaciones de las Normas Consolidadas para los Informes de los Ensayos Clínicos" (CONSORT). Es una lista de comprobación de 25 ítems que se consideran críticos para evaluar la transparencia y exactitud de las publicaciones, teniendo en cuenta diseño, ejecución, análisis y resultados de los estudios. La limitación más importante de los estudios fue el tamaño muestral y el no contar con estudios previos que permitan realizar una estimación válida para un cálculo de potencia, por lo cual la posibilidad de generalizar sus conclusiones es restringida. La fuente de financiación de los estudios y los conflictos de interés

Tabla 4. Evaluación de los estudios revisados con la "Escala de Jadad"

\begin{tabular}{|c|c|c|c|c|c|c|c|c|}
\hline Criterios & $\begin{array}{l}\text { Russ } \\
1994\end{array}$ & $\begin{array}{c}\text { Sonne } \\
1996\end{array}$ & $\begin{array}{l}\text { Roth } \\
1996\end{array}$ & $\begin{array}{c}\text { Bohus } \\
1999\end{array}$ & $\begin{array}{c}\text { Philipsen } \\
2004\end{array}$ & $\begin{array}{c}\text { Shmahl } \\
2012\end{array}$ & $\begin{array}{l}\text { Yovell } \\
2016\end{array}$ & $\begin{array}{c}\text { Martín-Blanco } \\
2017\end{array}$ \\
\hline $\begin{array}{l}\text { ¿Se describe el } \\
\text { estudio como } \\
\text { aleatorizado? }(*)\end{array}$ & NO & NO & NO & NO & NO & NO & SI & NO \\
\hline $\begin{array}{l}\text { ¿Se describe el } \\
\text { estudio como doble } \\
\text { ciego? }\left(^{*}\right)\end{array}$ & SI & NO & NO & NO & SI & SI & SI & NO \\
\hline $\begin{array}{l}\text { ¿Se describen las } \\
\text { pérdidas y retiradas } \\
\text { del estudio? }\left(^{*}\right)\end{array}$ & SI & NO & SI & SI & SI & SI & SI & SI \\
\hline $\begin{array}{l}\text { ¿Es adecuado } \\
\text { el método de } \\
\text { aleatorización? }\left({ }^{* *}\right)\end{array}$ & SI & NO & NO & NO & SI & SI & SI & NO \\
\hline $\begin{array}{l}\text { ¿Es adecuado el } \\
\text { método de doble } \\
\text { ciego? }\left(^{* *}\right)\end{array}$ & SI & NO & NO & NO & $\mathrm{SI}$ & $\mathrm{SI}$ & $\mathrm{SI}$ & NO \\
\hline Puntuación & 4 & -2 & -1 & -1 & 4 & 4 & 5 & -1 \\
\hline
\end{tabular}

$(*) \mathrm{Si}=1 / \mathrm{No}=0 ;(* *) \mathrm{Si}=1 / \mathrm{No}=-1$ 
de los autores sólo consta en 2 estudios: Schmahl (13) y Yovell (7). Los resultados de la evaluación de esta guía fueron comparables a los realizados por la Escala Jadad.

\section{Dificultades en la generalización de resultados}

Al revisar las bases de literatura científica, los estudios pertinentes son escasos, en muestras poblacionales muy pequeñas, con lapsos breves de tratamiento y seguimiento. Sólo la mitad de los estudios son de buena calidad metodológica.

Pensamos que estas dificultades pueden deberse a múltiples factores: la dificultad diagnóstica de este trastorno y la baja adherencia que podría traducirse en una amplia deserción de los estudios. Además, teniendo en cuenta que se trata de pacientes graves con poca o nula respuesta a tratamientos farmacológicos, podría implicar que las tasas de respuesta no sean equiparables a las de otros trastornos, obteniendo resultados que tal vez no tengan la contundencia que los profesionales de la salud desearíamos. Esto podría resultar en una falta de interés en continuar invirtiendo el tiempo y el esfuerzo que supone realizar investigación de calidad metodológica en grandes grupos poblacionales.

\section{Conclusiones}

Los resultados de la revisión de la literatura si bien no son concluyentes, aportan expectativas interesantes sobre posibles líneas de tratamiento.

De manera sintética podemos concluir que estos resultados parciales son los siguientes:

1. Naltrexona podría reducir los síntomas disociativos de desrealización, despersonalización, analgesia rela- cionada con el estrés, inmovilidad tónica y flashbacks.

2. Naltrexona, y tal vez nalmefeno, podrían reducir las ideas y conductas autolesivas sin fines suicidas (NSSI).

3. Nalmefeno podría mejorar los dominios clínicos del TLP.

4. Buprenorfina podría mejorar la ideación y conducta suicida del paciente límite y, en menor medida, la sintomatología depresiva.

5. Existe una correlación positiva que implica que el tratamiento a largo plazo es más eficaz que en monodosis.

6. Podría existir un efecto rebote ante la discontinuación abrupta de estos fármacos, por lo que se sugiere su retiro lento y gradual.

7. El perfil de pacientes que podría verse más beneficiado con estos fármacos es el subgrupo que cumpla la mayor cantidad de criterios para TLP y el que presenta analgesia relacionada al estrés.

Para poder arribar a conclusiones más firmes que permitan realizar una estimación válida para un cálculo de potencia, se necesitan más estudios de mejor calidad metodológica: con mayor validez interna, en muestras poblacionales más grandes, con control de variables de confusión que eviten los sesgos al realizar enmascaramiento doble ciego. Esperamos que la investigación continúe y en el futuro podamos contar con mayor evidencia y recursos terapéuticos para abordar la complejidad de estos pacientes.

\section{Conflicto de interés}

El autor declara no presentar conflictos de intereses respecto de este artículo.

\section{Referencias bibliográficas}

1. Bandelow B, Schmahl C, Falkai P, Wedekind D. Borderline personality disorder: a dysregulation of the endogenous opioid system? Psychological review. 2010;117(2):623-36.

2. Saper JR. Borderline personality, opioids, and naltrexone. Headache. 2000;40(9):765.

3. Soloff PH. Psychopharmacology of borderline personality disorder. The Psychiatric clinics of North America. 2000;23(1):169-92, ix.

4. Zhao $\mathrm{H}, \mathrm{Xu} \mathrm{H}, \mathrm{Xu} \mathrm{X}$. Effects of naloxone on the long-term potentiation of EPSPs from the pathway of Schaffer collateral to CA1 region of hippocampus in aged rats with declined memory. Brain research. 2004;996(1):111-6.

5. Russ MJ, Roth SD, Kakuma T, Harrison K, Hull JW. Pain perception in self-injurious borderline patients: naloxone effects. Biological psychiatry. 1994;35(3):207-9.

6. Butler S. Buprenorphine-Clinically useful but often misunderstood. Scandinavian journal of pain. 2013;4(3):148-52.
7. Yovell Y, Bar G, Mashiah M, Baruch Y, Briskman I, Asherov J, et al. Ultra-Low-Dose Buprenorphine as a Time-Limited Treatment for Severe Suicidal Ideation: A Randomized Controlled Trial. American journal of psychiatry. 2016;173(5):491-8.

8. Panksepp J, Watt D. Why does depression hurt? Ancestral primary-process separation-distress (PANIC/GRIEF) and diminished brain reward (SEEKING) processes in the genesis of depressive affect. Psychiatry. 2011;74(1):5-13.

9. Keating GM. Nalmefene: a review of its use in the treatment of alcohol dependence. CNS drugs. 2013;27(9):761-72.

10. Martin-Blanco A, Patrizi B, Soler J, Gasol X, Elices M, Gasol $\mathrm{M}$, et al. Use of nalmefene in patients with comorbid borderline personality disorder and alcohol use disorder: a preliminary report. International clinical psychopharmacology. 2017;32(4):231-4.

11. Philipsen A, Schmahl C, Lieb K. Naloxone in the treatment of acute dissociative states in female patients with borderline personality disorder. Pharmacopsychiatry. 2004;37(5):196-9. 
12. Bohus M, Landwehrmeyer G, Stiglmayr C, Limberger M Bohme R, Schmahl C. Naltrexone in the treatment of dissociative symptoms in patients with borderline personality disorder: an open-label trial. Journal of clinical psychiatry. 1999;60(9):598-603.

13. Schmahl C, Kleindienst N, Limberger M, Ludascher P, Mauchnik J, Deibler P, et al. Evaluation of naltrexone for dissociative symptoms in borderline personality disorder. International clinical psychopharmacology. 2012;27(1):61-8.

14. Sonne S, Rubey R, Brady K, Malcolm R, Morris T. Naltrexone treatment of self-injurious thoughts and behaviors. Journal of nervous and mental disease. 1996;184(3):192-5.

15. Roth AS, Ostroff RB, Hoffman RE. Naltrexone as a treatment for repetitive self-injurious behaviour:an open-label trial. Journal of clinical psychiatry. 1996;57(6):233-7.
16. Griengl H, Sendera A, Dantendorfer K. Naltrexone as a treatment of self-injurious behavior--a case report. Acta psychiatrica Scandinavica. 2001;103(3):234-6.

17. Thurauf NJ, Washeim HA. The effects of exogenous analgesia in a patient with borderline personality disorder (BPD) and severe self-injurious behaviour. European journal of pain (London, England). 2000;4(1):107-9.

18. McGee MD. Cessation of self-mutilation in a patient with borderline personality disorder treated with naltrexone. Journal of clinical psychiatry. 1997;58(1):32-3.

19. Stanley B, Sher L, Wilson S, Ekman R, Huang YY, Mann JJ. Non-suicidal self-injurious behavior, endogenous opioids and monoamine neurotransmitters. Journal of affective disorders. 2010;124(1-2):134-40. 